Projets

de paysage

\section{Projets de paysage}

Revue scientifique sur la conception et l'aménagement de l'espace

\section{9 | 2013}

Le paysage a t-il imposé sa loi ?

\title{
De l'optimalité pour la « ville verte »
}

Approche par analyse spatiale du réseau d'espaces ouverts niçois Optimality for the "green city" - Applying a spatial analysis approach on the open spaces network of Nice

\section{Sébastien Passel}

\section{OpenEdition}

\section{Journals}

Édition électronique

URL : http://journals.openedition.org/paysage/12713

DOI : 10.4000/paysage. 12713

ISSN : 1969-6124

\section{Éditeur :}

École nationale supérieure du paysage de Versailles-Marseille, Institut national des sciences appliquées Centre Val de Loire - École de la nature et du paysage, École nationale supérieure d'architecture et de paysage de Bordeaux, École nationale supérieure d'architecture et de paysage de Lille, Agrocampus Angers

\section{Référence électronique}

Sébastien Passel, « De l'optimalité pour la « ville verte » », Projets de paysage [En ligne], 9 | 2013, mis en ligne le 20 décembre 2013, consulté le 21 novembre 2020. URL : http://journals.openedition.org/ paysage/12713; DOI : https://doi.org/10.4000/paysage.12713

Ce document a été généré automatiquement le 21 novembre 2020.

Projets de paysage 


\title{
De l'optimalité pour la «ville verte »
}

\author{
Approche par analyse spatiale du réseau d'espaces ouverts niçois \\ Optimality for the "green city" - Applying a spatial analysis approach on the \\ open spaces network of Nice
}

\section{Sébastien Passel}

1 La ville de Nice (341 000 habitants en 2009 et plus d'un million dans son aire urbaine ${ }^{1}$ ), située au Sud-Est de la France, sur la Côte d'Azur, est devenue au fil des ans une destination attractive, notamment au niveau touristique, subissant en conséquence de nombreuses pressions sur le foncier disponible. Selon les données issues de la base d'occupation du sol (OccSol) du Centre régional de l'information géographique (Crige) de la région PACA (figure 1), l'emprise du tissu urbain a augmenté de $16 \%$ entre 1990 et 2006 (moyenne nationale $6 \%$ ), principalement dans les vallées à l'ouest de la ville, au détriment des surfaces agricoles (- 30 \% sur la même période ; en France, - $3 \%$ ). Dans ce contexte, les élus de la ville doivent considérer la conservation et la valorisation des espaces naturels et agricoles comme étant une nouvelle perspective dans la maîtrise de son développement territorial. L'élaboration du plan local d'urbanisme (PLU), en 2010, a permis l'autopromotion de Nice au rang de «ville verte de la Méditerranée » (Ville de Nice, 2010).

2 Si l'idée de « ville verte » renvoie généralement à celle d'espaces urbains végétalisés, il n'en demeure pas moins qu'instaurer de la nature en ville relève du défi pour l'aménageur, tant les deux termes paraissent antagonistes : "Par définition, la ville est un espace opposé à la nature. Ainsi, dans un sens large, on nomme nature tout ce qui n'est pas la ville» (Brunet et al., 2009, p. 345). Pourtant, il y existe bien des espaces interstitiels, non bâtis, non imperméabilisés - les « espaces ouverts " - pouvant servir de pistes pour repenser l'urbanité (Banzo, 2009, p. 5). La notion d'espace ouvert désigne « une portion d'espace privé ou public dont la fonction principale est de répondre à des usages divers: de l'activité récréative à la protection des ressources naturelles, en passant par la contemplation paysagère, souvent réduite à la mise en scène d'un cadre qu'il faut préserver de tout aménagement inopportun » (Banos et Sabatier, 2010, p. 8). 
Figure 1. Évolutions des occupations du sol à Nice entre 1990 et 2006

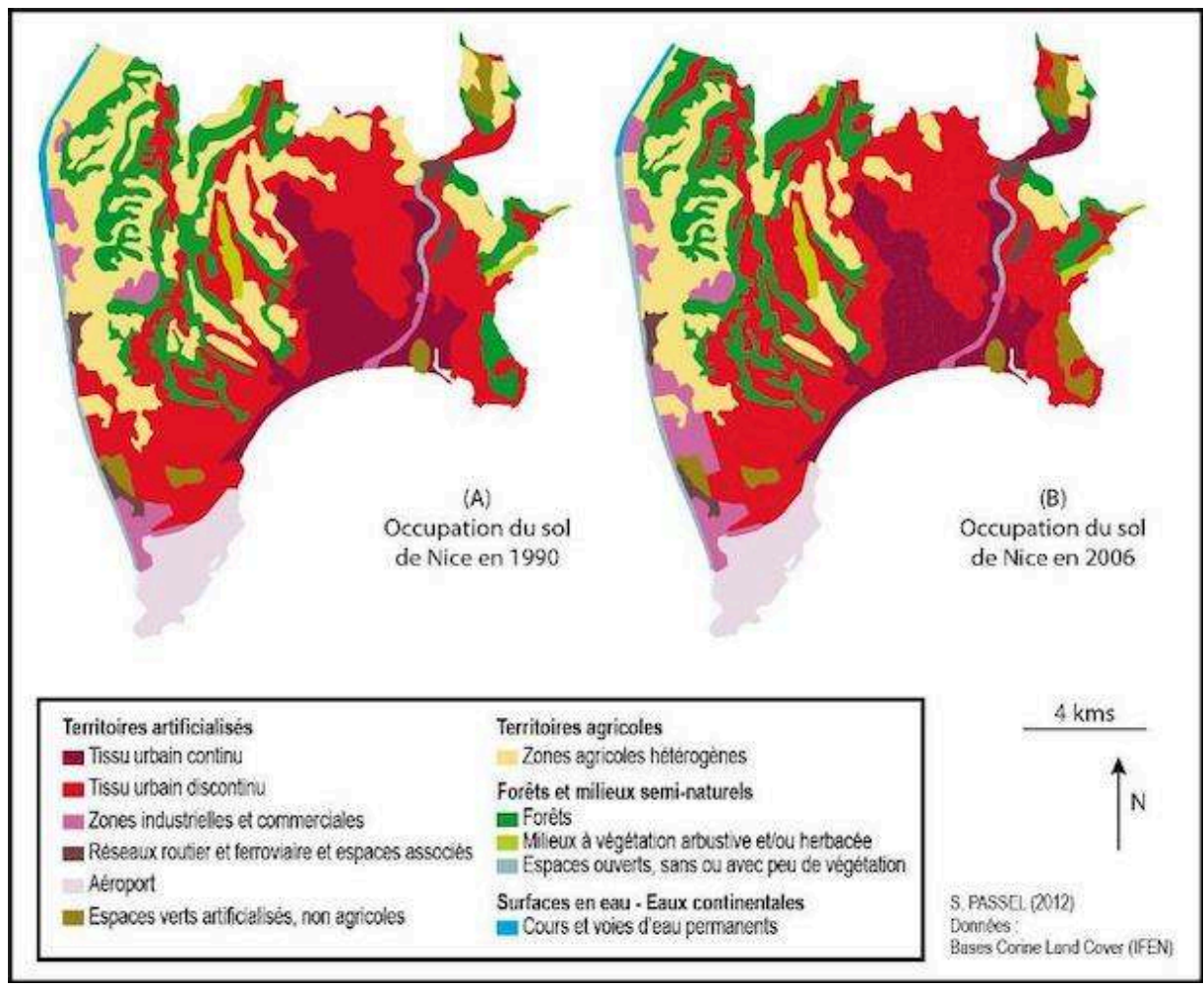

3 Cette valorisation progressive des "aménités paysagères et récréatives " que sont les espaces ouverts entraîne une diversification des usages et des pratiques sur un même lieu (Banos et Sabatier, 2010). L'espace ouvert, au-delà de ses perspectives paysagères, doit également être accepté comme celui de l'ouverture au public: en l'envisageant dans une dynamique de publicisation, l'espace ouvert peut être distingué comme une nouvelle forme d'espace public (Banzo, 2009). Or, si les espaces publics sont considérés comme la matrice de fond, ou encore le catalyseur de la vie urbaine (Jacobs, 1961), alors nous posons l'hypothèse qu'une mise en réseau des espaces ouverts publics (par exemple, les parcs et autres jardins publics) avec les boisements, ainsi que les espaces agricoles et naturels, participerait au bon fonctionnement d'une grande ville comme Nice. La structure urbaine sera ainsi considérée comme optimale lorsque le niveau d'équité y sera maximisé. Cette équité est relative à la façon dont les avantages induits par les espaces ouverts sont répartis entre les agents (Bailly et al., 1995).

L'objectif du présent article est de proposer une approche quantitative en utilisant des méthodes et des outils issus de l'analyse spatiale, afin de "décrire une disposition particulière de certains objets, leur organisation dans l'espace, repérer des structures, expliquer une localisation par d'autres" (Pumain et Saint-Julien, 2005, p.5). Des techniques issues de la morphologie mathématique serviront à la modélisation des informations et autres phénomènes géographiques permettant de déceler des connectivités potentielles des espaces ouverts, dans le but de concevoir un réseau qui contribuerait à optimiser la répartition spatiale des espaces ouverts. Le recours à l'analyse spatiale se justifie par le fait que nous considérons l'espace géographique tel un construit "par lequel le géographe représente le réel et cherche à l'expliquer » (Voiron, 1993, p. 24). 


\section{"Ville verte » et mise en réseau des espaces ouverts}

5 Considérant les perspectives d'aménagement actuelles, donner un sens à la «ville verte » n'est pas sans équivoque. En réalité, la « ville verte » ne possède pas de modèle concret : par conséquent, elle pourrait être définie de plusieurs manières (Vernay et al., 2010, cités par Mallet, 2012). Ainsi, dans son plan local d'urbanisme, la ville de Nice ne mentionne jamais ce qui est entendu par l'emploi du terme de "ville verte ", même s'il en ressort quatre orientations distinctes: " préserver et valoriser un paysage et un environnement exemplaires", "se loger et vivre ensemble", "mieux circuler et se déplacer autrement », " affirmer Nice comme métropole internationale » (Ville de Nice, 2010). De par ces objectifs relativement convenus, la «ville verte » telle que conçue par la ville de Nice ne permet pas d'en définir son sens concret.

6 D'une manière générale, si une ville peut être assimilée «à un système décomposable en sous-systèmes, on doit y trouver des réseaux d'interactions » (Dupuy, 1983, p. 9). La dynamique d'interaction des espaces ouverts générerait la diffusion des aménités induites par les espaces ouverts (Saint-Julien, 1985), notamment auprès des populations vivant dans des espaces très artificialisés (figure 8 ). En effet, les citadins sont très demandeurs de «nature » à proximité de leur logement, la présence de végétal étant considérée tel un gage de satisfaction de qualité de vie dans un quartier (Boutefeu, 2007). Il est donc essentiel d'établir une connexité du réseau, c'est-à-dire une liaison entre les espaces ouverts, afin de permettre divers échanges et circulations. Aussi, la tendance des aménageurs est de promouvoir les continuités vertes au sein de l'urbain, appelées greenways (ou "voies vertes » en français). La littérature à ce sujet distingue les corridors écologiques (pour le passage de la faune et de la flore) des corridors récréatifs, davantage portés comme "voies de communication autonomes réservées aux déplacements non motorisés, développées dans un souci d'aménagement intégré valorisant l'environnement et la qualité de vie " (Association européenne des voies vertes, 2000). Les urbanistes parlent alors de tisser une "toile verte, entre les populations et la terre, entre les parcs publics, les espaces naturels, patrimoniaux et les autres espaces ouverts " (Toccolini et al., 2006, p. 100). Il s'agit en outre d'améliorer la connectivité du système, en proposant des chemins alternatifs pour développer un réseau plus complexe, qui permettrait la multiplicité du nombre et des types de liens possibles entre les espaces ouverts du territoire. Ainsi, ceux-là seraient pleinement intégrés dans la quotidienneté des citadins et constitueraient un tout avec le reste de la ville (et non plus envisagés comme simples espaces résiduels).

7 À partir de ces considérations, nous avons établi une cartographie des espaces ouverts de Nice ${ }^{2}$ (figure 2). Les espaces intégrés au tissu urbain ont été distingués grâce à un croisement de données des différents espaces ouverts avec la couche SIG issue de la base OccSol, représentant le tissu artificialisé de la ville de Nice. Cette catégorie d'espaces ouverts se définit de par son accessibilité facilitée (par exemple, pour les bois et forêts, par les quelques sentiers qui longent les vallons), au cœur d'îlots denses (notamment pour les parcs et jardins publics), avec des fréquentations plus importantes et des usages multifonctionnels (Boutefeu, 2007). Les espaces ouverts extérieurs au tissu urbain se situent sur du foncier limitant naturellement la constructibilité et l'accessibilité (reliefs vallonnés, terrains inondables...), avec un mitage de faible densité. La voirie arborée est également intégrée à la typologie, faisant référence aux axes dont le gabarit permet l'aménagement de "voies vertes ${ }^{3}$ ", et sert à 
la constitution du maillage recherché de ce que nous pouvons considérer comme un système d'espaces ouverts.

Figure 2. Le sytème d'espaces ouverts existant sur la ville de Nice

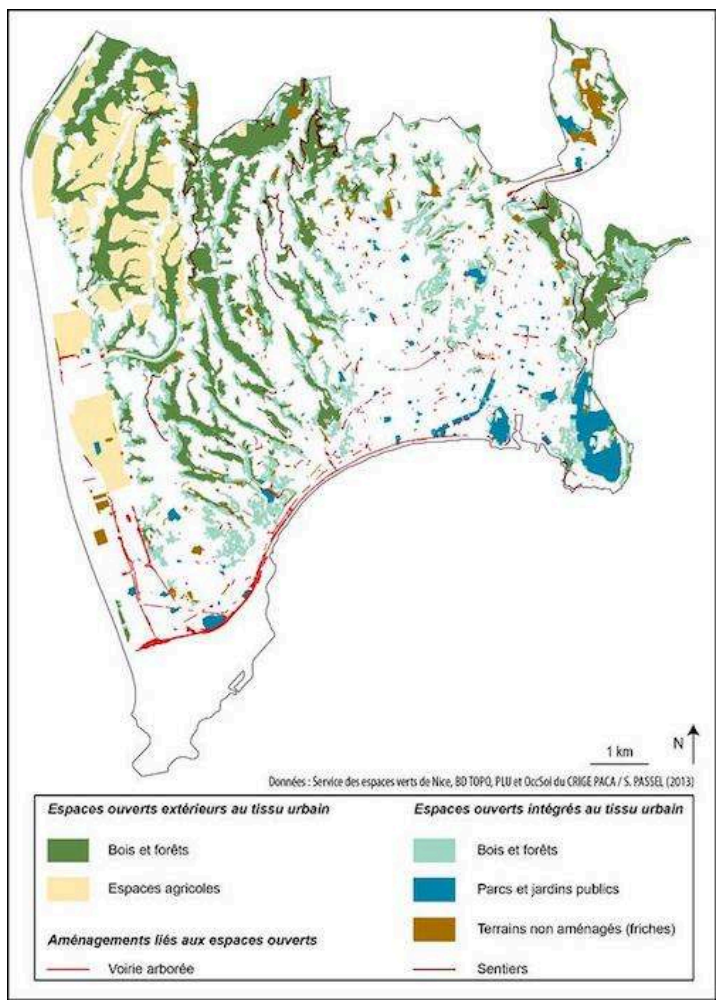

\section{Analyse spatiale et analyse d'images: quelles connectivités potentielles pour « Nice, ville verte »?}

Cette partie propose une analyse de modélisation de la connectivité (au sens spatial du terme) des espaces ouverts. Les algorithmes issus de la morphologie mathématique permettront de localiser les différents espaces à enjeux de la ville de Nice, notamment en matière de fragmentation des espaces ouverts. L'idée est, d'une part, de repérer les discontinuités spatiales, c'est-à-dire des ruptures majeures dans le paysage urbain; d'autre part, de détecter des structures potentielles favorables à la mise en réseau des espaces ouverts.

éliser les dynamiques d'un espace suppose de prendre en compte son potentiel, en reliant formes et structures spatiales aux processus : appréhender la morphologie des éléments d'une ville et leurs relations est indissociable de l'étude d'un paysage urbain (Allain, 2004). En prenant parti pour l'analyse spatiale, « on cherchera notamment les liens qui unissent les phénomènes ponctuels ou linéaires (villes, réseaux, littoral, frontière) aux phénomènes de surface (peuplement, espaces agricoles, naturels, bâtis), afin de découvrir les corrélations spatiales » (Voiron, 1993, p. 28).

La démarche adoptée est voisine de celle utilisée en modélisation: il s'agit du traitement d'images, avec, comme finalité, de faire émerger d'elle une signification. La morphologie mathématique est alors une aide à l'interprétation des structures et des dynamiques spatiales, car elle combine les apports de la cartographie, où les formes 
sont là pour représenter les structures décelées, et ceux issus des modèles graphiques, où les formes doivent, en outre, révéler les mécanismes en jeu (Voiron, 1993). Pour traiter des phénomènes géographiques, les séquences de traitement auront comme finalité de "dégager les structures spatiales les plus significatives et de rattacher une forme visible à des propriétés générales ou bien aux processus qui lui ont donné naissance» (ibid., p. 27). Le logiciel de traitement d'images par algorithmes de morphologie mathématique "Micromorph» constitue ici l'outil privilégié de nos travaux et sera utilisé pour appréhender les formes de structuration de l'espace générées par le système d'espaces ouverts de la ville de Nice.

\section{Les transformations ensemblistes et morphologiques}

11 L'analyse spatiale par la morphologie mathématique permet d'établir des séquences de transformation des images dans le but d'en faire naître de nouvelles, comportant plus de significations. La démarche consiste donc à simplifier progressivement les images jusqu'à la mise en évidence d'une forme ou d'une structure caractéristique (Voiron, 1995a). En outre, la morphologie mathématique s'appuie principalement sur la théorie des ensembles; des transformations dites ensemblistes comme l'union ou encore l'intersection permettent de sélectionner et de combiner des éléments appartenant à deux images distinctes $\mathrm{X}$ et $\mathrm{Y}$ (Voiron, 1993).

Les transformations morphologiques agissent sur des images binaires, et « s'effectuent au moyen d'un élément structurant : comme une sorte de sonde envoyée dans l'image dans le but de recueillir des renseignements morphologiques" (ibid., p. 61). Sur une image binaire, l'érosion et la dilatation sont considérées comme des transformations morphologiques élémentaires (figure 3). L'érosion s'effectue au moyen d'un élément structurant ayant une forme et une taille choisies par le chercheur. La transformation conduit à une diminution des contours de la structure initiale et à la disparition des objets de taille inférieure à l'élément structurant. La dilatation se traduit par un grossissement des contours, un comblement des trous et une connexion entre les formes proches. Ces opérations ne sont pas linéaires: une érosion pratiquée sur la forme dilatée ne permet pas de retrouver l'image de départ (Voiron, 1995a).

Figure 3. Quelques transformations morphologiques élémentaires

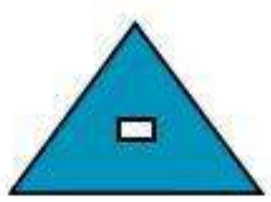

Image A

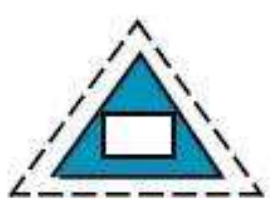

Image A erodée

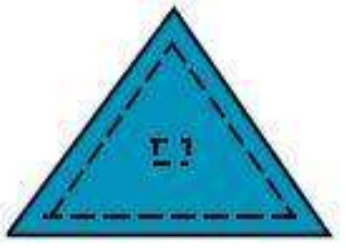

Image $A$ dilatee

Source : Voiron, 1995b.

Ces deux transformations de base peuvent être combinées de manière différente. Ainsi, une ouverture s'effectue en deux temps : érosion de l'image de départ puis dilatation de l'image érodée. Une fermeture correspond à la séquence inverse: dilatation puis érosion. Une ouverture réalisée à partir d'un cercle ou d'un hexagone supprime les petites composantes et gomme les irrégularités alors qu'une fermeture bouche les trous et réunit les composantes proches (figure 4). 
14 Si l'on réitère la séquence de transformations choisies avec un élément structurant de taille croissante, on obtient un tamisage des composantes. Des mesures sont ensuite effectuées après chaque transformation, "d'où le terme de granulométrie pour effectuer ce type de traitement. Dans le cas de la granulométrie par fermeture, l'information structurale est relative à la distance, car ce ne sont plus les composantes mais l'espace situé entre elles qui est tamisé ; au fur et à mesure que la taille de la fermeture augmente, les composantes se rattachent les unes aux autres jusqu'à n'en former plus qu'une seule» (Voiron, 1995b, p. 18). Les différentes transformations indiquent alors la distance ou le temps mis par les composantes pour se raccorder ainsi que le chemin emprunté.

Figure 4. Transformations par ouverture et fermeture
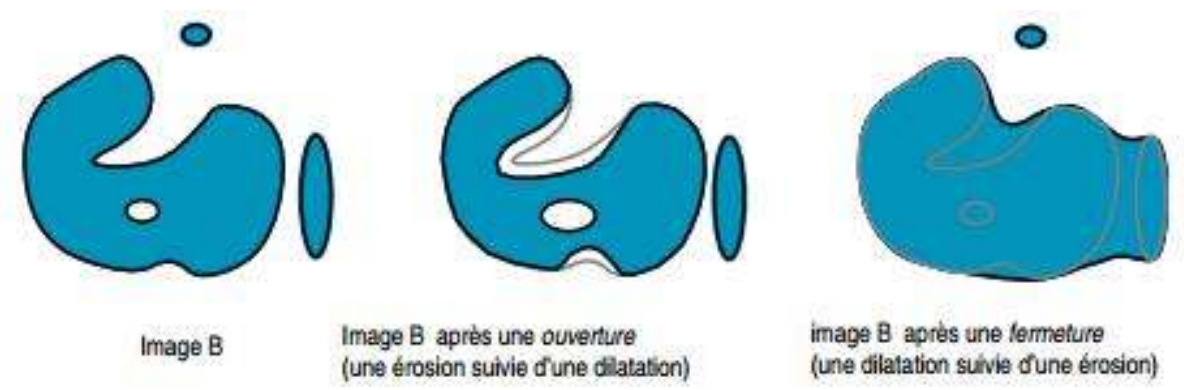

Source : Voiron, 1995b.

\section{Granulométrie par fermeture des espaces ouverts}

15 Toutes les transformations d'images sont effectuées par algorithmes de fermeture, à l'aide d'hexagones de taille croissante. En premier lieu, de façon à pouvoir effectuer nos traitements de morphologie mathématique, une exportation de la couche SIG de la figure 2 sur Micromorph nous a permis de traiter l'image en format binaire, en comblant les éventuels résidus (ou "trous») engendrés par la pixellisation des éléments: pour les traitements de morphologie mathématique, les pixels blancs prendront la valeur « 1 » et les pixels noirs la valeur « $0 »$ (figure 5). 
Figure 5. Image binaire servant de base aux traitements : le système d'espaces ouverts niçois

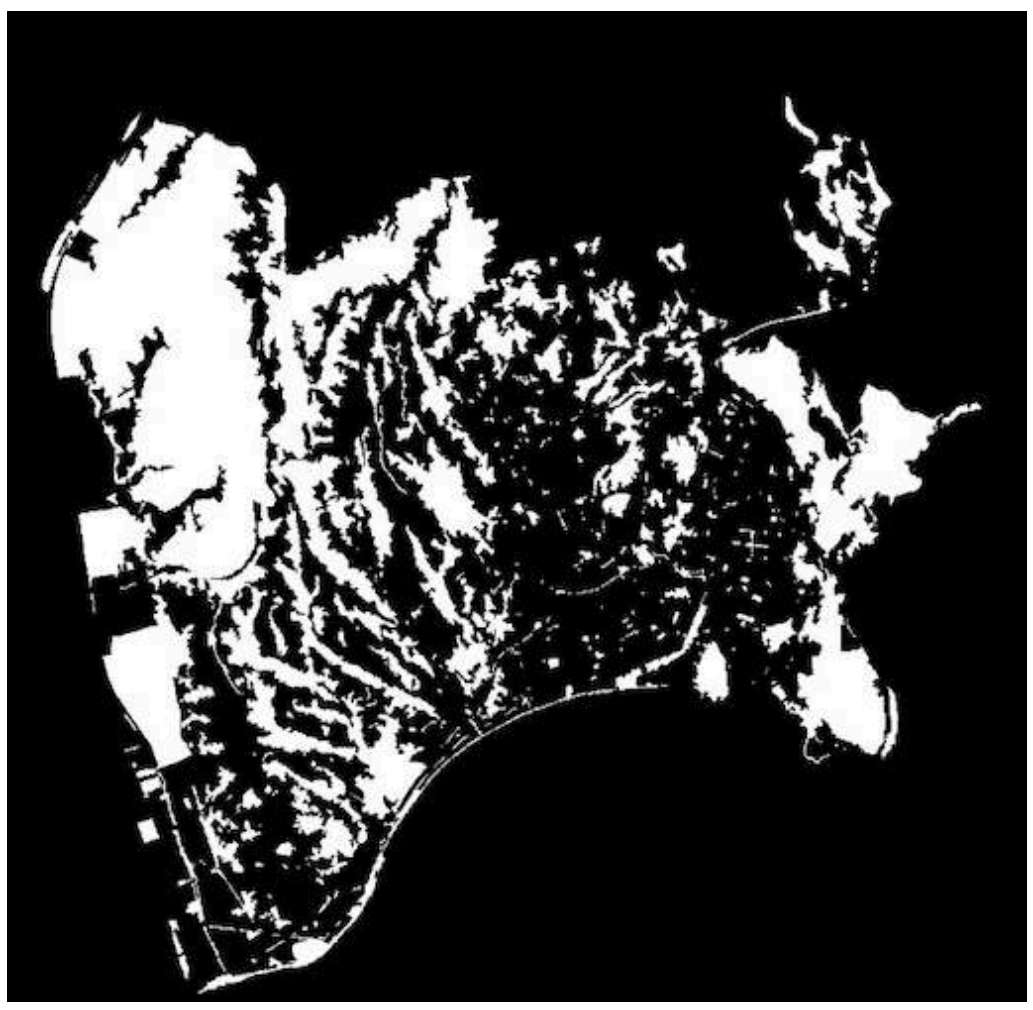

16 La granulométrie par algorithme de fermeture apporte des informations sur l'espacement des éléments composant le système, ainsi que sur les différents cheminements privilégiés à la mise en réseau des espaces les plus proches. Ainsi, il faudra attendre la fermeture de taille 13 afin que la totalité des éléments soit agrégée en une seule et même composante (figure 7). Cette situation s'apparente au maximum de connexité pour les espaces ouverts de Nice, où chacun d'entre eux est relié à une autre composante au minimum.

Figure 6. Courbe de connexité issue des fermetures successives sur l'image des espaces ouverts

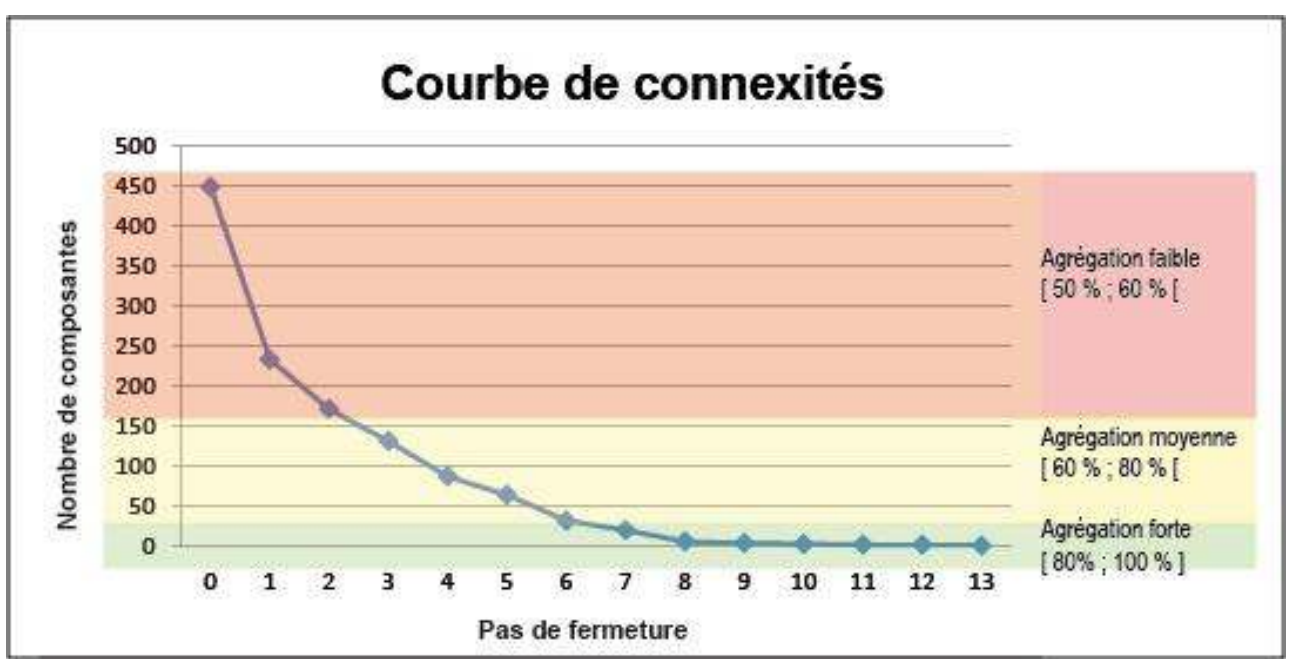

17 L'allure générale de la courbe de connexité (figure 6) détermine différentes classes dans l'agrégation des espaces ouverts. Ainsi, à partir du pas de fermeture de taille 7 , le 
nombre de composantes n'évolue que très peu, la grande majorité des espaces ouverts niçois étant mise en réseau : ce palier devient alors le seuil à atteindre dans les aménagements de connexité du système.

En situation actuelle, c'est-à-dire au pas de fermeture 0, les espaces ouverts sont agrégés à hauteur de $51 \%$. Cette situation peut s'expliquer par le comblement des «trous» sur l'image binaire (figure 5), révélant les «zones tampons» initiales permettant de connecter les entités les plus proches les unes des autres. Ainsi, les vastes espaces ouverts extérieurs au tissu urbain, situés aux franges nord-ouest et est de la ville, contribuent fortement à expliquer ce taux de connexité. Par opposition, les parcs et jardins publics ne représentent que 6 des $51 \%$ de la mise en réseau des espaces ouverts en situation initiale.

19 Sur la figure 7, les coalescences d'éléments de la fermeture de taille 3 suivent une logique d'alignement plus ou moins continu: les espaces naturels et agricoles des franges est et ouest de la ville sont déjà bien agrégés; en revanche, ils ne sont connectés à l'urbain qu'en prenant notamment appui sur la promenade des Anglais. On remarque que les parcs et jardins publics manquent de liens de connexité : les bois et forêts intégrés au tissu artificialisé permettent cependant l'agrégation progressive de ces espaces fragmentés à la composante principale. Avec les fermetures de taille supérieure, les connexions se multiplient, raccordant les espaces ouverts entre eux, dans la composante principale (appelée «le continent»). D'autres espaces se sont agrégés de par leur proximité, mais ne sont pas reliés au continent car la taille de la « zone tampon » (l'espace joignant deux entités voisines) n'est pas encore suffisante ; on peut qualifier ces éléments telles des « îles » par opposition au « continent ». Sur la fermeture de taille 7 , nous pouvons observer une segmentation du continent selon une logique nord-sud, correspondant aux grands axes routiers localisés sur des reliefs relativement plats, ou dans les vallons. Sur ces voies, la morphologie des bâtis est de forme linéaire : juxtaposés de manière à former une ligne continue, ils contribuent au morcellement du système.

Lorsque la totalité des espaces ouverts est reliée au continent, comme cela est le cas à partir de la fermeture de taille 13, la connexité est égale à $100 \%$. En revanche, il convient davantage de s'intéresser aux "vides ultimes ", signalant les trous majeurs de la mise en réseau des éléments. Ces éléments sont nuisibles à la connectivité optimale du système, dans le sens où la segmentation qui y est générée ne permet pas d'assurer la multiplicité des liens possibles entre les espaces ouverts de la ville. Il faudra ainsi prolonger l'analyse jusqu'à un pas de fermeture de taille 24 afin d'obtenir $100 \%$ de connectivité, ne laissant aucun espace fragmenté dans la ville. 
Figure 7. Images obtenues avec différents pas de fermeture
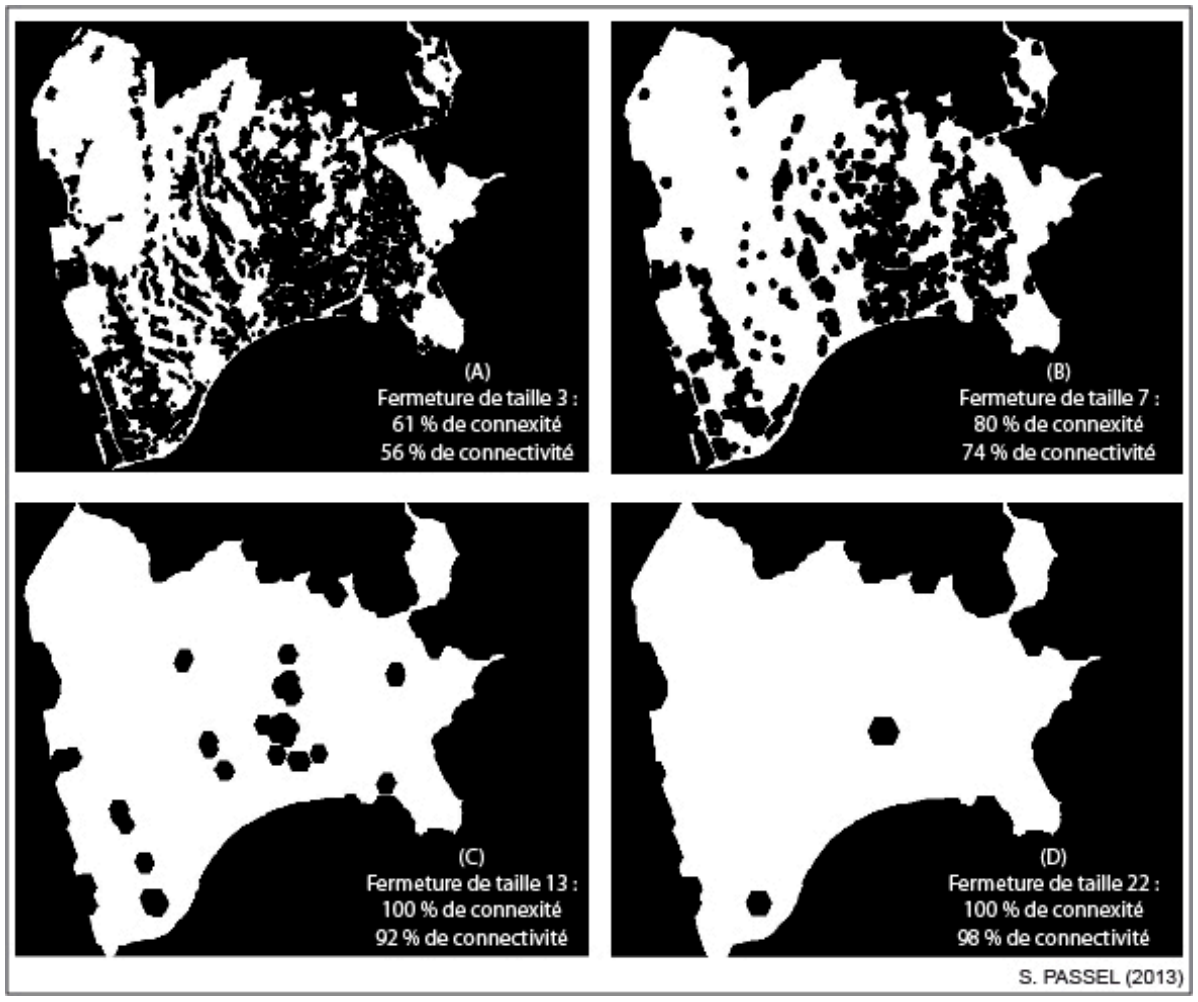

21 Deux types de vides, révélant les espaces de fragmentation principaux (c'est-à-dire les secteurs de la ville où la connexité du système est la plus difficile), peuvent être observés (figure 8). D'une part, les quartiers dits " populaires ", à forte densité de bâtis, à dominante générale de logements sociaux (Roquebillière et Pasteur: Nice-est, Las Planas et Saint-Sylvestre : Nice-nord, Magnan, Saint-Augustin et Les Moulins : Nice-sudouest). D'autre part, des espaces à forte artificialisation peuvent être décelés, notamment en raison d'une emprise importante des infrastructures industrielles (quartier du Port, marché d'intérêt national, zone commerciale de Lingostière). Le quartier de la gare centrale SNCF est le plus fragmenté de la ville, ne se rattachant au continent qu'à l'itération finale (24), où connexité et connectivité sont égales à $100 \%$. Quant au quartier de Saint-Isidore, autrefois résidentiel, situé sur la plaine du Var, il fait l'objet d'une immense restructuration ${ }^{4}$, notamment avec la construction en cours d'un stade de football et de grandes surfaces commerciales sur des terres qui étaient agricoles. Ces espaces sont contraignants pour la mise en réseau du système d'espaces ouverts parce qu'ils sont très artificialisés - ou le deviennent progressivement (dans la plaine du Var), et l'enjeu d'aménager des «zones tampons » est ici majeur, de manière à faciliter l'accessibilité aux espaces ouverts pour les personnes parcourant ces lieux dans le cadre de leur quotidienneté. 
Figure 8. Les principaux espaces de fragmentation de la ville de Nice

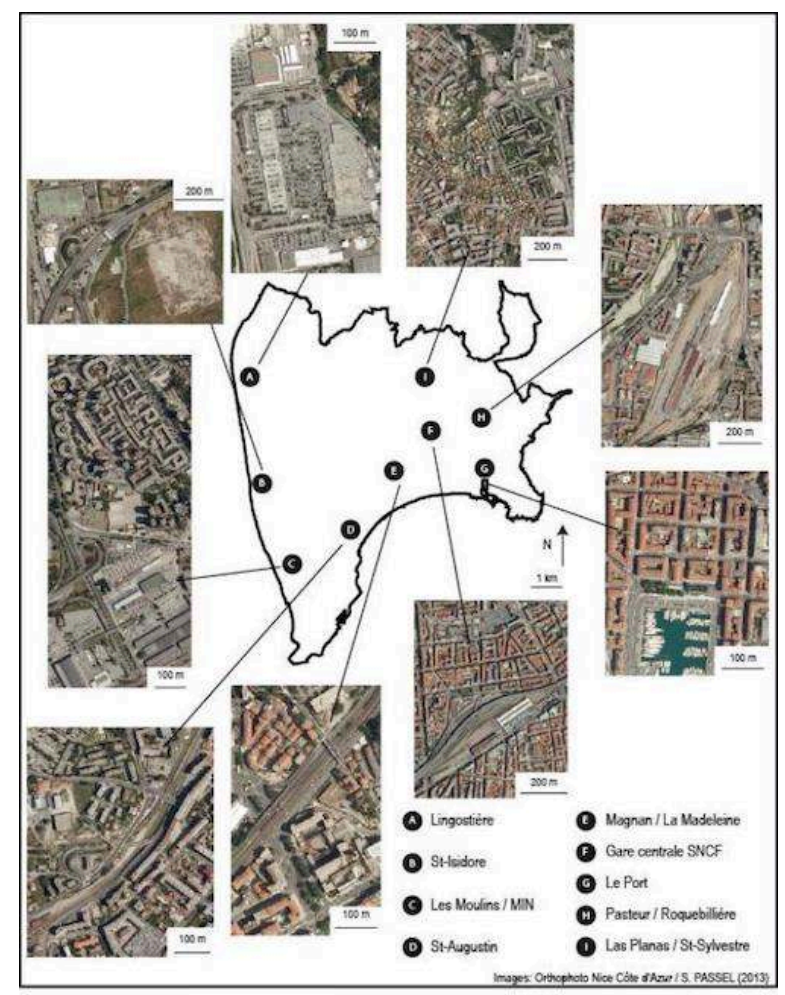

\section{Traitements morphologiques au maillage du système}

Les images issues des algorithmes de fermeture ont certes l'avantage de permettre la détection d'espaces majeurs de fragmentation, il n'en demeure pas moins que les «zones tampons» créées par les algorithmes s'établissent sur un espace euclidien, et ne prennent pas en compte les contraintes de l'existant, notamment le tissu artificialisé. Où aménager, de façon concrète, ces zones nécessaires à l'établissement d'un maillage du système d'espaces ouverts? Il s'agit d'intégrer une nouvelle contrainte à nos analyses de morphologie mathématique, de façon à rendre les résultats intelligibles.

Les «zones tampons " ainsi détectées seront intersectées avec le réseau routier de la ville (hors autoroute et pénétrantes urbaines) afin de localiser les tronçons à aménager en priorité pour améliorer la mise en réseau des espaces ouverts (figure 9). Lorsque toutes les composantes de l'image seront agrégées, il sera alors possible d'analyser la situation de connectivité maximale à prendre en compte pour la ville de Nice. 
Figure 9. Méthodologie d'analyse morphologique pour augmenter la connectivité du système d'espaces ouverts
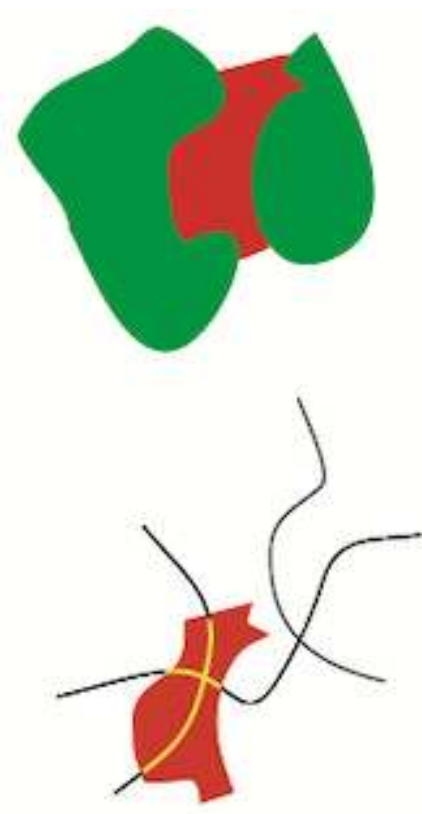

\section{Etape 1 :}

Transformation de granulométrie par fermeture morphologique des espaces verts

\section{Etape 2:}

Intersection de la nouvelle composante obtenue ("zone tampon", en rouge) avec le réseau routier

\section{Etape 3 :}

Dètextion des tronçons à aménager en priorité (en jaune) pour améliorer la connectivité des espaces verts

Source : Sébastien Passel, 2013.

La figure 10 détaille les opérations menées afin d'obtenir les résultats souhaités. Tout d'abord, une fermeture (de taille 6, pour l'exemple) est effectuée sur l'image initiale du système d'espaces ouverts (A). Afin d'extraire les zones tampons de l'image obtenue par la fermeture (B), nous procédons à un traitement par algorithme de différence ensembliste: dans ce type de transformation, nous sélectionnons uniquement les éléments de (B) n'appartenant pas à (A). L'ajout d'une contrainte supplémentaire à l'analyse implique le passage de la couche SIG du réseau routier de la ville de Nice en image binaire (D), par un protocole identique à l'image (A). Les zones tampons révélées sur l'espace euclidien sont ensuite intersectées avec (D). L'image finale (F) consiste en la superposition des routes considérées par l'analyse morphologique du réseau connectant les espaces ouverts au système initial (correspondant à $47 \%$ de connectivités potentielles) avec l'image (E), de façon à déterminer les tronçons de routes à recomposer en "voies vertes ». Ainsi, $22 \%$ de connectivité supplémentaire permettraient de relier une majorité d'espaces ouverts au sein de la ville (tableau 1). 
Tableau 1. Connexité et connectivité des espaces ouverts aux différents pas de fermeture

\begin{tabular}{cccc}
$\begin{array}{c}\text { Pas de } \\
\text { fermeture }\end{array}$ & Nombre d'éléments & \% connexité & \% connectivité \\
\hline 0 & 448 & 50,9 & 47,0 \\
\hline 1 & 233 & 54,7 & 50,4 \\
\hline 2 & 171 & 57,7 & 53,2 \\
\hline 3 & 131 & 61,0 & 56,2 \\
\hline 4 & 87 & 65,4 & 60,3 \\
\hline 5 & 64 & 70,3 & 64,7 \\
\hline 6 & 32 & 74,7 & 68,8 \\
\hline 7 & 20 & 80,1 & 73,8 \\
\hline 8 & 6 & 84,1 & 77,5 \\
\hline 9 & 4 & 88,4 & 81,5 \\
\hline 10 & 3 & 92,2 & 85,0 \\
\hline 11 & 2 & 95,0 & 87,5 \\
\hline 12 & 2 & 97,8 & 90,2 \\
\hline 13 & 1 & 100,0 & 92,2 \\
\hline 22 & 1 & 100,0 & 97,8 \\
\hline 24 & 1 & 100,0 & 100,0 \\
\hline
\end{tabular}

Figure 10. Protocole de détection des tronçons routiers à aménager en zone tampon
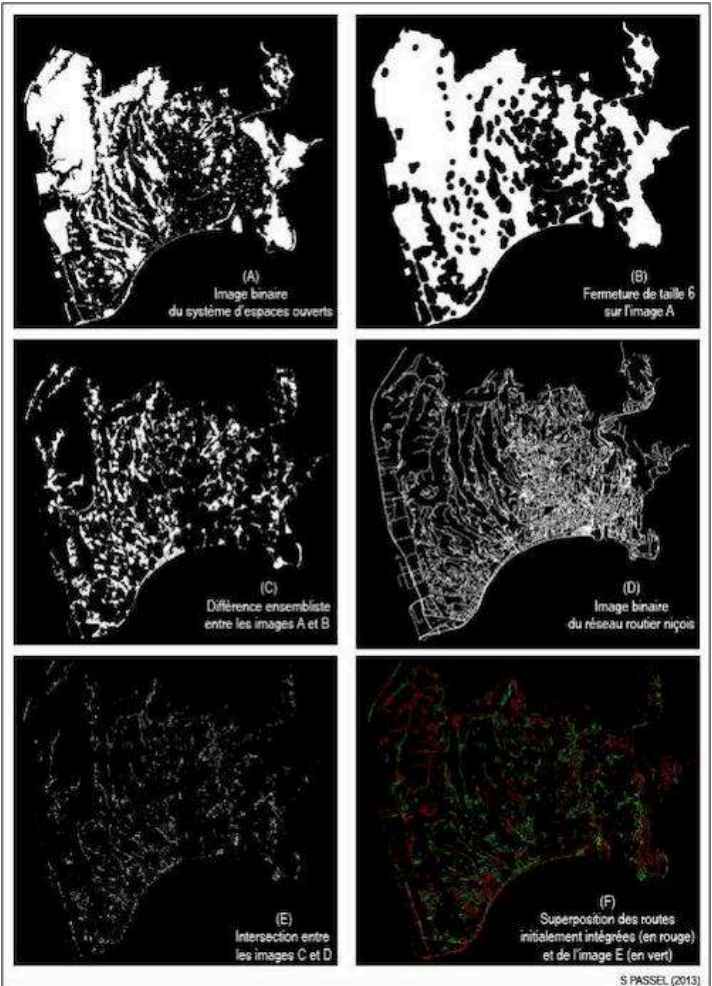

Si l'on décompose l'image finale (F) obtenue en figure 10, huit secteurs présentent des structures spatiales distinctes (figure 11). Le système d'espaces ouverts de Nice se 
caractérise par une très forte dynamique littorale est-ouest: ainsi, la majorité des tronçons à aménager en zones tampons permet de relier les espaces ouverts du tissu urbain via la promenade des Anglais. Dans ce cas, l'aménagement de tels tronçons sur la plaine du Var et les collines à l'ouest de la ville inscrirait les espaces agricoles et naturels propices à la biodiversité, qui composent ces secteurs, dans une potentielle dynamique de publicisation. Les grands parcs publics, supérieurs à un hectare, semblent également jouer un rôle prépondérant dans la perspective d'une mise en réseau des espaces ouverts. En effet, ils peuvent non seulement créer des interfaces entre les sous-systèmes urbains identifiés, mais également permettre aux populations vivant dans des quartiers très artificialisés, comme ceux du cœur de ville ou des quartiers nord et est, de pouvoir bénéficier d'une meilleure accessibilité à ces grandes superficies regroupant «des qualités fonctionnelles et symboliques appréciées et recherchées des citadins" (Boutefeu, 2007).

De ce point de vue, le protocole d'analyse morphologique détectant des tronçons à aménager pour améliorer la connectivité du système d'espaces ouverts niçois permet d'orienter le processus décisionnel des urbanistes car il localise les lieux potentiellement générateurs de connectivité. Il s'agit alors de relier les espaces agricoles et naturels à la dynamique littorale de la ville, et de favoriser l'accessibilité des quartiers denses aux grands parcs urbains, qui permettent l'interaction entre les différents sous-systèmes. Ainsi, des aménagements tels que la "coulée verte ${ }^{5}$ " pourraient être considérés comme des points nodaux du système d'espaces ouverts niçois, dans la mesure où ils connecteraient, d'une part, les espaces ouverts extérieurs au tissu urbain mais bien reliés au littoral et, d'autre part, les espaces ouverts pleinement intégrés à l'urbain, notamment grâce à l'axe nord-sud entre la vieille ville et le quartier résidentiel de Cimiez. Les petites aspérités des tronçons permettent également de détecter les espaces favorables à la mise en réseau des squares et autres jardins publics, en particulier au sein des sous-systèmes à forte densité urbaine (quartiers centraux, nord et est en particulier). 
Figure 11. Structures et dynamiques du système d'espaces ouverts à aménager

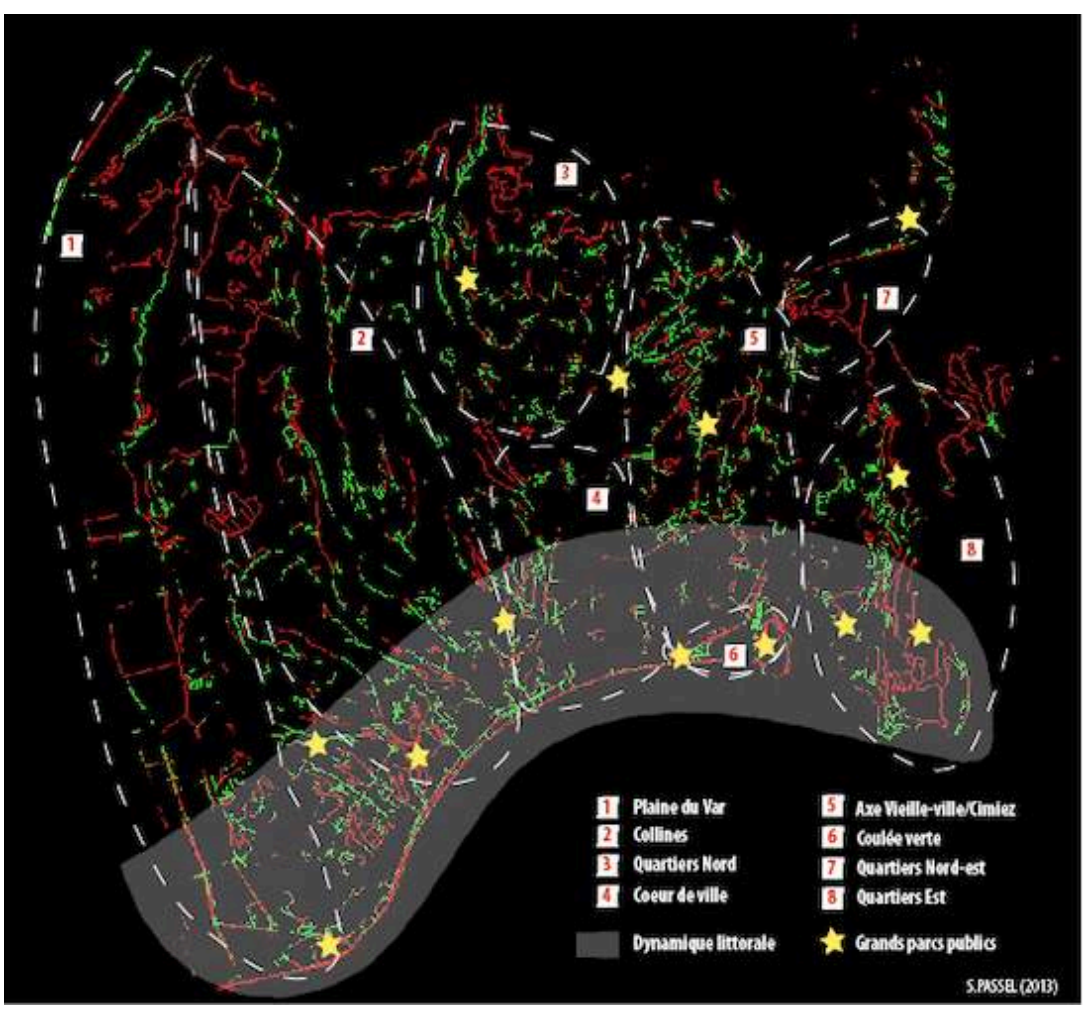

\section{Conclusion}

La morphologie mathématique apparaît comme une méthode intéressante à la détection de structures et de dynamiques spatiales : en révélant les espaces à enjeux niçois, les résultats permettent de localiser les lieux de la ville qui ne sont pas innervés par les espaces ouverts, pour aider à la décision quant aux aménagements qui devront être réalisés.

Dans notre conception de l'optimalité pour la «ville verte", nous suggérons que la structure urbaine soit maximisée selon son niveau d'équité, c'est-à-dire la façon dont la répartition des avantages induits par les espaces ouverts est réalisée entre les agents. Cette répartition peut toutefois s'envisager sous différents aspects en interaction : si la morphologie mathématique nous a apporté des éléments de réponse concernant la structure spatiale existante, il n'en demeure pas moins que les aménités générées par les espaces ouverts seront perçues différemment selon le contexte urbain dans lequel le citadin vit. Par conséquent, suivant les quartiers, un espace public aura une ambiance profondément différente (Bassand et al., 2001).

L'originalité de la recherche réside par le fait que nous abordions les espaces ouverts dans une " problématique du phénomène urbain » (ibid., p. 1), mettant en exergue leurs processus morphologiques, tout en considérant l'ensemble des pratiques et des représentations citadines qui y sont associées comme éléments fondateurs d'une construction territoriale (Blanc, 1995, p. 15). Cette recherche se positionne ainsi à l'interface d'une géographie spatiale d'un côté, et sociale de l'autre. Les prochaines étapes de la recherche concerneront l'imbrication des rapports sociaux et spatiaux que les hommes nouent avec leur territoire (Di Méo, 2001), et le rôle des espaces ouverts 
dans les représentations de l'espace par les citadins. De ce fait, la prochaine démarche sera résolument orientée vers une approche dite qualitative, basée sur une analyse discursive et une recherche d'images mentales, révélant le témoignage des citadins visà-vis de leur(s) environnement(s).

\section{BIBLIOGRAPHIE}

Allain, R., Morphologie urbaine. Géographie, aménagement et architecture de la ville, Paris, Armand Colin, coll. «U », 2004, 254 p.

Association européenne des voies vertes, « Déclaration de Lille », 11-12 septembre 2000, URL : http://www.aevv-egwa.org/site/1Template1.asp?

DocID $=21 \& v 1 \mathrm{ID}=\&$ RevID $=\&$ namePage $=\&$ pageParent

Bailly, A., Baumont, C., Huriot, J.-M., Sallez, A., Représenter la ville, Paris, Economica, 1995, 107 p.

Banos, V., Sabatier, B., « Les espaces périurbains non bâtis en France : entre publicisation "urbaine" et privatisation "rurale" ?", Articulo - Journal Urban Research, 2010, hors-série nº 3 , 20 p., URL: http://articulo.revues.org/1524.

Banzo, M., «L'espace ouvert pour une nouvelle urbanité », mémoire d'habilitation à diriger des recherches, université de Bordeaux 3, vol. 3, 2009, 202 p.

Bassand M., Compagnon, A., Joye, D., Stein, V., Vivre et créer l'espace public, Lausanne, Presses polytechniques et universitaires romandes, 2001, $223 \mathrm{p}$.

Blanc, N., « La nature dans la cité », thèse de doctorat de l'université Paris I, 1995, 392 p.

Boutefeu, E., « Le paysage dans tous ses états. La nature en ville : des enjeux paysagers et sociétaux ", Géoconfluences, 2007, URL : http://geoconfluences.ens-lyon.fr/doc/transv/paysage/ PaysageViv.htm.

Brunet, R., Ferras, R., Thery, H., Les Mots de la géographie. Dictionnaire critique (1 ${ }^{\text {re }}$ édition, 1993), Paris, Reclus-La Documentation française, 2009, p. 345.

Di Méo, G., Géographie sociale et territoires, Paris, Nathan Université, 2001, 317 p.

Dupuy, G., « Villes, systèmes et réseaux : le rôle historique des techniques urbaines ", Réseaux, vol. $2 \mathrm{n}^{\circ} 4,1983$, p. 3-23.

Jacobs, J., Déclin et Survie des grandes villes américaines, Bruxelles, Mardaga, 1961, 433 p.

Mallet, J., « Les villes vertes : analyse de leurs réalisations et propositions de recommandations pour leur développement », essai de maîtrise universitaire en environnement, université de Sherbrooke (Canada), 2012, $122 \mathrm{p}$.

Mathieu, N. et Guermond, Y., La Ville durable, du politique au scientifique, Versailles, Éditions Quæ, 2005,285 p.

Pumain, D., et Saint-Julien, T., L'Analyse spatiale, localisations dans l'espace, Paris, Armand Colin, coll. «Cursus », 2005, 167 p.

Saint-Julien, T., La Diffusion spatiale des innovations, Montpellier, GIP Reclus, 1985, 37 p. 
Toccolini, A., Fumagalli, N., Senes, G., « Greenways planning in Italy: the Lambro River Valley greenway system », Landscape and Urban Planning, $\mathrm{n}^{\circ} 76,2006$, p. 98-111.

Ville de Nice, Plan local d'urbanisme. Synthèse du rapport de présentation, 2010, 19 p.

Voiron, C., Analyse spatiale et analyse d'images, Montpellier, GIP Reclus, 1995a, 190 p.

Voiron, C., « Morphologie d'un semis de villes européennes », Mappemonde, n 4, 1995b, p. 17-21.

Voiron, C., « Espace, structures et dynamiques régionales : l'arc méditerranéen », Revue d'analyse spatiale quantitative et appliquée du laboratoire Raoul Blanchard, université de Nice-Sophia Antipolis, $\mathrm{n}^{\circ} 33-34,1993,349 \mathrm{p}$.

\section{NOTES}

1. Chiffres provenant du recensement général de population de l'Insee.

2. Les jardins privés n'ont pas été intégrés dans la typologie, compte tenu de l'absence de bases de données mises à notre disposition.

3. Les voies inaccessibles aux piétons comme les pénétrantes, certaines routes nationales ou encore les autoroutes urbaines ont été retirées de cette classe.

4. Le quartier Saint-Isidore est situé au cœur du périmètre de l'opération d'intérêt national (OIN) "plaine du Var », qui s'étend sur 15 communes, représentant quelque 10000 hectares de foncier potentiellement exploitables.

5. La «coulée verte ", renommée "promenade du Paillon », est présentée par la ville de Nice comme "un parc urbain de 12 hectares en cœur de ville, une magnifique promenade verte offerte aux piétons ». Le site a été inauguré le 26 octobre 2013.

\section{RÉSUMÉS}

Aujourd'hui, quatre Français sur cinq vivent en ville. L'urbanisation généralisée de ces dernières décennies a conduit à une crise profonde de l'urbain, se manifestant par une dégradation des conditions de vie dans la plupart des quartiers (Mathieu et Guermond, 2005). La question d'une nouvelle urbanité se pose à travers la dynamique de répartition des espaces ouverts, qui contribueraient à l'optimisation de la structure d'une ville. Une étude d'analyse spatiale par morphologie mathématique sera menée pour détecter les espaces à enjeux liés à la structure existante des espaces ouverts de la ville de Nice.

Today, four French people out of five live in cities. The widespread urbanization in recent decades has led to a deterioration of living conditions in most urban areas (Mathieu and Guermond, 2005). The question of a new urbanity arises through the dynamics of open space distribution that contributes to the optimization of the city structure. A spatial analysis study using mathematical morphology will be conducted to detect the potentialities of Nice's open spaces system. 
INDEX

Mots-clés : analyse spatiale, espaces ouverts, morphologie mathématique

Keywords : spatial analysis, open spaces, mathematical morphology

\section{AUTEUR}

\section{SÉBASTIEN PASSEL}

Titulaire d'un master 2 recherche en analyse spatiale et d'un master 2 professionnel en urbanisme et aménagement du territoire, Sébastien Passel est actuellement doctorant en géographie et aménagement du territoire au sein de l'UMR Espace de Nice (université de NiceSophia Antipolis) sur une recherche concernant les rapports entre espaces bâtis et espaces ouverts urbains, ainsi que les perceptions et pratiques citadines qui en découlent. sebastien.passel[at]unice[dot]fr 